\title{
Determinação do limiar anaeróbio pela variabilidade da frequência cardíaca de pacientes com DPOC durante exercício em cicloergômetro
}

\author{
Determining anaerobic threshold through heart rate variability \\ in patients with COPD during cycloergometer exercise
}

\author{
Flávia Cristina Rossi Caruso ${ }^{[a]}$, Michel Silva Reis ${ }^{[b]}$, Ana Cristina Barroso Siqueira ${ }^{[c]}$, Marli Gardim ${ }^{[d]}$, \\ Aparecida Maria Catai ${ }^{[e]}$, Audrey Borghi-Silva ${ }^{[f]}$ \\ [a] Fisioterapeuta, mestranda em Fisioterapia Cardiovascular, Laboratório de Fisioterapia Cardiopulmonar, Núcleo de \\ Pesquisa em Exercício Físico, Departamento de Fisioterapia, Universidade Federal de São Carlos (UFSCar), São Carlos, \\ SP - Brasil, e-mail: fla.rossi@hotmail.com \\ [b] Fisioterapeuta, pós-doutorando em Fisioterapia Cardiovascular pela Universidade Federal de São Carlos (UFSCar), \\ docente do curso de Fisioterapia Cardiorrespiratória da Universidade Federal do Rio de Janeiro (UFRJ), Rio de Janeiro, \\ RJ - Brasil, e-mail: michelsilre@hotmail.com \\ [c] Fisioterapeuta, mestre em Medicina pela Universidade Federal de São Paulo (Unifesp), São Paulo, SP - Brasil, e-mail: \\ anacristinabarroso@yahoo.com.br \\ [d] Fisioterapeuta, especialista em Geriatria pela Universidade Federal de São Carlos (UFSCar), São Carlos, SP - Brasil, e-mail: \\ marligardim@gmail.com \\ [e] Docente do curso de Fisioterapia da Universidade de Federal de São Carlos (UFSCar), São Carlos, SP - Brasil, e-mail: \\ mcatai@ufscar.br \\ [f] Fisioterapeuta, docente do curso de Fisioterapia Cardiovascular da Universidade Federal de São Carlos, (UFSCar), São \\ Carlos, SP - Brasil, e-mail: audrey@ufscar.br
}

\section{Resumo}

Introdução: A Doença Pulmonar Obstrutiva Crônica (DPOC) é caracterizada por alterações estruturais do parênquima pulmonar, acarretando manifestações sistêmicas. Além disso, esses pacientes podem apresentar marcada alteração do controle autonômico cardíaco. Nesse contexto, estudos investigando a variabilidade da frequência cardíaca (VFC) em pacientes com DPOC durante a realização de exercício físico em cicloergometro têm sido pouco explorados. Objetivo: Determinar o limiar anaeróbio por meio da VFC com 
o propósito de estabelecer parâmetros de avaliação e prescrição da intensidade de exercício desses pacientes em cicloergômetro. Materiais e métodos: Foram avaliados oito pacientes do sexo masculino com diagnóstico de DPOC com idade média de 69,5 \pm 7,6 anos. A frequência cardíaca foi analisada no repouso e em diferentes intensidades do exercício. 0 teste foi realizado em um cicloergômetro e consistiu em um período de aquecimento de quatro minutos em uma potência mínima. Foram, também, realizados degraus com potência inicial de 4W, com acréscimos de 5 em $5 \mathrm{~W}$, até que o paciente atingisse o limiar anaeróbio. Resultados: Os pacientes que apresentaram maiores valores de $\mathrm{VEF}_{1}$ apresentaram maiores potências no cicloergômetro. Houve redução significativa da VFC durante o exercício físico se comparado ao repouso sentado $\mathrm{p}<0,05$. Conclusão: Os pacientes estudados apresentaram um severo descondicionamento físico reafirmado pela impossibilidade determinada pelo LA de $50 \%$ da amostra.

Palavras-chave: Limiar anaeróbio. Exercício físico. DPOC. Frequência cardíaca.

\begin{abstract}
Introduction: Chronic Obstructive Pulmonary Disease (COPD) is characterized by structural alterations of lung parenchyma resulting in systemic manifestations. These patients may have marked change in cardiac autonomic control. In this context, studies investigating heart rate variability (HRV) in patients with COPD during physical exercise cycloergometers have been little explored. Objective: To determine the anaerobic threshold through HRV in order to establish parameters of evaluation and prescription of exercise intensity in these patients on a cycloergometer. Materials and methods: Eight male patients diagnosed with COPD, mean age $69.5 \pm 7.6$ years were studied. Heart rate was analyzed at rest and at different intensities of exercise. The test was performed on a cycloergometer and consisted of a warm-up period of four minutes at a minimum power. Steps were performed with initial power of $4 \mathrm{~W}$, with increments of $5 \mathrm{in} 5 \mathrm{~W}$, until the patient reached the anaerobic threshold. Results: Patients that presented higher values of FEV $V_{1}$ showed greater powers during the cycloergometer. Additionally, there was significant reduction in HRV during exercise compared to rest sitting $p$ $<0.05$. Conclusion: Patients had a severe physical deconditioning reaffirmed the impossibility determined by the anaerobic threshold of $50 \%$ of the sample.
\end{abstract}

Keywords: Anaerobic threshold. Physical exercise. COPD. Heart rate.

\section{Introdução}

A doença pulmonar obstrutiva crônica (DPOC) é caracterizada por um quadro de obstrução progressiva ao fluxo aéreo, na maioria dos casos, relacionado a uma resposta inflamatória dos pulmões $(1,2)$. 0 volume expiratório forçado durante o primeiro segundo da manobra expiratória $\left(\mathrm{VEF}_{1}\right)$ e a relação $\mathrm{VEF}_{1} / \mathrm{CVF}$ (capacidade vital forçada) são importantes índices para o diagnóstico e classificação do grau de obstrução pulmonar em portadores de $\operatorname{DPOC}(3,4)$. A intolerância ao exercício físico (EF) é um problema comum em pacientes com DPOC. Essa patologia produz diminuição na qualidade de vida dos pacientes por ela acometidos (5).

Nesse sentido, vários estudos afirmam benefícios da atividade física na reabilitação do indivíduo com DPOC (6-10). Apesar de o EF ter um papel importante no tratamento desses pacientes, melhorando sua capacidade física, reduzindo a dispneia (11), aumentando a força muscular respiratória (12) e periférica (13), melhorando, assim, a qualidade de vida (5), considera-se que a intensidade de exercício proposto é um fator determinante na magnitude dos efeitos atingidos com o treinamento. Isso porque muitos pacientes com obstrução moderada-grave não conseguem sustentar esforços com intensidade suficiente para que ocorram modificações fisiológicas benéficas, por apresentarem limitação muscular periférica e ventilatória (13).

A integração dos sistemas cardiovascular, respiratório e muscular constitui um complexo e sofisticado mecanismo de geração de energia durante o exercício físico. Para avaliar a integração cardiopulmonar durante o exercício físico, o limiar de anaerobiose (LA ou LA1) tem sido um importante índice de desempenho 
aeróbio (14). Além disso, o limiar de compensação respiratória (LCR ou LA2) tem sido útil na determinação do desempenho em intensidades near-máximas (14).

A análise das respostas ventilatórias e metabólicas obtidas em testes de esforço físico durante um teste cardiopulmonar (TCP) refletem a respiração celular dos músculos periféricos, porém com certo atraso (15). Esse atraso pode ser explicado pelo retardo no circuito dos gases para ocupar todo o volume disponível em seu trajeto musculoalveolar até serem captados ao nível da boca e, também, em virtude da difusibilidade do oxigênio e do dióxido de carbono (15).

Outra ferramenta útil na determinação dos limiares é a resposta da frequência cardíaca (FC). Estudos prévios mostraram que a aplicação de modelos matemáticos aplicados aos dados da produção de dióxido de carbono $\left(\mathrm{VCO}_{2}\right)$ mostrou forte relacionamento com os dados de FC em indivíduos saudáveis (16) na determinação do LA. A validade da determinação do LA pela FC configura-se como importante estratégia para avaliação da capacidade funcional por meios mais simples e economicamente viáveis.

Dessa forma, o objetivo desse estudo foi determinar o LA por meio da resposta da FC e de sua variabilidade, com o propósito de estabelecer parâmetros de avaliação e prescrição da intensidade do exercício físico desses pacientes em protocolos descontínuos em cicloergômetro.

\section{Materiais e métodos}

\section{Pacientes}

Foram triados para este estudo oito pacientes do sexo masculino com diagnóstico clínico de DPOC. Todos foram submetidos à anamnese completa (história clínica atual e pregressa, hábitos de vida, valores espirométricos recentes e medicamentos em uso), e avaliação física composta por medidas antropométricas. Posteriormente, os pacientes foram submetidos à avaliação clínica, com o objetivo de caracterizar a coexistência de outras patologias; e ao teste ergométrico (esforço máximo ou sintoma limitante) realizado por uma cardiologista para descartar a presença de isquemia do miocárdio ou respostas inadequadas da FC, pressão arterial (PA) e do sinal eletrocardiográfico que contraindicasse a participação no trabalho.

Todos os pacientes foram informados sobre os procedimentos experimentais a que seriam submetidos, assim como sobre a evidência do caráter não invasivo dos testes. Os pacientes que concordaram em participar do estudo assinaram um termo de consentimento livre e esclarecido, de acordo com as normas do Conselho Nacional de Saúde sob Parecer n. 238/05.

\section{Critérios de inclusão}

Foram incluídos neste estudo pacientes com diagnóstico clínico de DPOC apresentando $\mathrm{VEF}_{1}<60 \%$, com idade superior a 50 anos, na ausência de exacerbações agudas da doença, estáveis clinicamente, ex-tabagistas e que não se submetiam à reabilitação cardiorrespiratória.

\section{Critérios de exclusão}

Foram excluídos deste estudo pacientes com doenças respiratórias associadas (tuberculose, bronquiectasia, asma), hipertensão arterial não controlada, cardiopatias graves, deficit de compreensão, processos neoplásicos, sequelas neurológicas associadas ou problemas osteoarticulares que impedissem a realização do protocolo proposto.

\section{Procedimentos gerais}

Os testes foram realizados no Núcleo de Pesquisa da Instituição. Os experimentos foram realizados no mesmo período do dia considerando as influências circadianas do organismo. Para a realização dos testes, os pacientes foram orientados a: comparecer com roupas confortáveis; não ingerir bebidas alcoólicas e/ou estimulantes 24 horas antes dos testes; fazer a última refeição (leve) pelo menos duas horas antes do início do protocolo; não realizar atividade física extenuante no dia anterior; evitar o uso de medicamentos, como broncodilatadores inalatórios; e, principalmente, manter as demais medicações habituais (se houvesse).

Além disso, os pacientes foram orientados para que, durante a execução dos protocolos, não falassem desnecessariamente com os avaliadores e informassem sobre quaisquer alterações percebidas em seu estado geral, como mal-estar, tontura ou surgimento de outros sintomas que impossibilitassem a continuidade dos testes. 


\section{Protocolo experimental}

\section{Protocolo I - Condição de repouso}

na posição sentada

Teve como objetivo avaliar a VFC durante o repouso na posição sentada. Inicialmente, os pacientes foram posicionados na postura supina para que fossem realizadas a tricotomia e a limpeza da pele, bem como o posicionamento dos eletrodos - derivação MC5 (polo negativo - eletrodo vermelho no manúbrio; polo positivo - eletrodo amarelo no quinto espaço intercostal esquerdo na linha axilar anterior e o eletrodo neutro preto no rebordo costal direito). Posteriormente, solicitou-se que o paciente sentasse em uma cadeira, onde o sinal eletrocardiográfico foi captado, por 15 minutos, em um monitor cardíaco de um canal (TC -500, Ecafix). Acoplado ao monitor havia um conversor analógico digital (Lab - PC + National Instruments) que constituiu numa "interface" entre aquele e o microcomputador (Pentium III). A partir desse acoplamento, o sinal analógico do eletrocardiograma (ECG) era convertido em valores binários para o acesso no microcomputador, por meio de um programa de processamento dos sinais digitalizados. Dessa maneira, a FC e os intervalos $\mathrm{R}-\mathrm{R}$ (iR-R) foram captados e armazenados batimento a batimento, permitindo análises posteriores dos dados. Ao início e final da coleta foram aferidas PA, utilizando-se um esfignomanômetro (BD $\left.{ }^{\circledR}\right)$ e estetoscópio (Littman ${ }^{\circledR}$ ).

Protocolo II - Teste de esforço físico dinâmico em degraus descontínuos em cicloergômetro (TEFDD)

Teve como objetivo determinar a resposta da FC e de sua variabilidade, bem como a contribuição dos componentes do sistema nervoso autônomo simpático e parassimpático no incremento da FC induzida pelo EF em diferentes intensidades de esforço físico.

Esse teste foi conduzido em um cicloergômetro (Quinton Corival 400/Load ${ }^{\circledR}$ ) e consistiu de um período de aquecimento de quatro minutos em uma potência mínima ( $4 \mathrm{~W})$. Foram realizados degraus com potência inicial de $4 \mathrm{~W}$, em seguida $5 \mathrm{~W}$ e, a partir de então, acréscimos de $5 \mathrm{em} 5 \mathrm{~W}$, até que o paciente atingisse a intensidade correspondente ao LA. Após a determinação do LA, foi testada uma última intensidade com potência de $5 \mathrm{~W}$ acima do mesmo. A FC e os iR-R foram registrados batimento a batimento (conforme descrito anteriormente) durante um minuto antes do início do esforço, quatro minutos em cada estágio de esforço e no primeiro minuto de recuperação após o degrau testado. A orientação em todas as intensidades de exercício foi a manutenção da velocidade em 60 rotações por minuto (rpm). A saturação periférica de $\mathrm{O}_{2}\left(\mathrm{SpO}_{2}\right)$ foi verificada continuamente durante todo o experimento, por meio de um oxímetro de pulso (Takaoka Oxifast 9064), e a PA foi mensurada anteriormente ao primeiro minuto, nos últimos 30 segundos do estágio de esforço e após a recuperação.

No fim de cada estágio de esforço, os pacientes foram questionados sobre o grau de percepção da intensidade de esforço por meio da escala de Borg (CR-10) (17). Entre um nível de esforço e outro, os pacientes aguardaram em repouso sentado o retorno aos valores basais da FC e PA. Para os pacientes que apresentassem dessaturação após o término dos degraus, seria ofertado $\mathrm{O}_{2}$ por meio de uma máscara facial conectada a um umidificador e um cilindro de oxigênio.

Durante o período em exercício (estabilização da FC), foi selecionado o trecho entre 120 e 270 segundos de dados de FC captados durante o EF, e analisados pela aplicação de um modelo matemático e estatístico semiparamétrico a partir de uma rotina específica desenvolvida para este fim, utilizando-se o aplicativo SPLUS (versão 4.5 Professional Release 2 for Windows, 1998). No primeiro nível de esforço, quando ocorreu a desestabilização da FC, ou seja, deflexão positiva da curva de distribuição da FC, foi identificada como o LA, indicando, assim, predomínio da atuação simpática sobre o nó sinoatrial.

\section{Análise estatística}

Os dados obtidos foram submetidos ao teste de normalidade (teste de Shapiro-Wilks) e, por apresentarem distribuição não normal, foi selecionado um teste estatístico não paramétrico Friedman com post hoc de Dunn pareado $(\mathrm{p}<0,05)$ para relacionar a FC média (FCMed) e os índices RMSSD (raiz quadrada da somatória do quadrado da diferença entre os iR- $R$ consecutivos do registro do ECG, dividido pelo número de iR-R em um tempo determinado menos um) e RMSM (raiz quadrada da somatória do quadrado das diferenças dos valores individuais em relação ao valor médio, dividido pelo número de iR-R da série de dados selecionados). As análises foram realizadas 
nas situações de repouso sentado, e durante o EF dinâmico (no LA e acima do LA). Os gráficos foram apresentados em box-plots, com medianas, $1^{\circ}$ e $3^{\circ}$ quartis e valores mínimos e máximos.

\section{Resultados}

A Tabela 1 mostra a idade, as características antropométricas, o índice preditivo do $\mathrm{VEF}_{1}, \mathrm{SpO}_{2}$ em repouso e a necessidade de suplementação de $\mathrm{O}_{2}$ dos pacientes estudados, em médias e desvios-padrão. Nela podemos observar que os pacientes estavam na faixa etária entre 56 e 76 anos e apresentaram valores como peso, altura, índice de massa corpórea (IMC) e $\mathrm{SpO}_{2}$ em repouso com baixas variações.

\section{Análise das potências próximas ao LA}

Na Tabela 2, são apresentadas as intensidades em que foram obtidos o LA, a primeira potência que o antecedeu e a intensidade logo acima dele. Lá se pode perceber também que, na nossa amostra, não foi possível obter a potência abaixo do LA de todos os pacientes, visto que metade dos pacientes apresentaram inclinação positiva no gráfico do comportamento da FC já na intensidade mínima do cicloergômetro (4W).
Relação entre $\mathrm{LA}_{\text {e }} \mathrm{VEF}_{1}$

A Tabela 2 indica que os pacientes cujo LA obtido foi próximo a $4 \mathrm{~W}$ também apresentaram os menores índices de $\mathrm{VEF}_{1}$. Exceção feita ao paciente 2, que, apesar de ter obtido a intensidade do LA próxima a $20 \mathrm{~W}$, apresentou $\mathrm{VEF}_{1}$ de $42 \%$ do predito (semelhante ao paciente 8, que apresentou LA em $4 \mathrm{~W}$ ). Paralelamente, podemos verificar que os pacientes que apresentaram os três maiores índices de $\mathrm{VEF}_{1}$ conseguiram realizar intensidades de EF superiores aos $4 \mathrm{~W}$, com destaque para o paciente 5 , que apresentou o maior valor para $\mathrm{VEF}_{1}(55,2 \%$ do predito) e a maior intensidade do LA (50W).

Análise de FCMed e VFC no domínio do tempo

A Figura 1 ilustra a FCMed obtida em repouso sentado e durante o EF dinâmico nos níveis de potência do LA e acima do LA. Observamos que os valores de FCMed foram significativamente maiores nos níveis de potência correspondentes ao LA e acima do LA, em comparação à condição de repouso.

0 índice RMSSD obtido em repouso e durante as duas potências de EF dinâmico - na intensidade do LA e acima do LA estão representados na Figura 2.

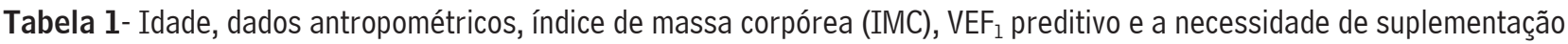
de $\mathrm{O}_{2}$ dos pacientes

\begin{tabular}{|c|c|c|c|c|c|c|c|}
\hline Pacientes & $\begin{array}{l}\text { Idade } \\
\text { (anos) }\end{array}$ & $\begin{array}{c}\text { Massa } \\
(\mathrm{kg})\end{array}$ & $\begin{array}{l}\text { Estatura } \\
(\mathrm{cm})\end{array}$ & $\begin{array}{c}\text { IMC } \\
\left(\mathrm{kg} / \mathrm{m}^{2}\right)\end{array}$ & $\begin{array}{l}\text { VEF }_{1} \\
(\%)\end{array}$ & $\begin{array}{c}\mathrm{SpO}_{2} \text { em } \\
\text { repouso (\%) }\end{array}$ & Suplementação $\mathbf{O}_{2}$ \\
\hline 1 & 75 & 73,00 & 167 & 26,17 & 36,0 & 91 & $\mathrm{Sim}^{\star}$ \\
\hline 2 & 65 & 68,00 & 172 & 22,97 & 42,0 & 89 & Não \\
\hline 3 & 63 & 68,50 & 166 & 24,85 & 27,0 & 90 & Não \\
\hline 4 & 56 & 49,20 & 164 & 18,29 & 32,5 & 91 & Não \\
\hline 5 & 76 & 79,50 & 172 & 26,85 & 55,2 & 96 & Não \\
\hline 6 & 69 & 82,00 & 171 & 28,08 & 44,0 & 93 & Não \\
\hline 7 & 77 & 60,50 & 160 & 23,63 & 50,5 & 96 & Não \\
\hline 8 & 75 & 64,00 & 174 & 21,06 & 42,0 & 92 & Não \\
\hline Média & 69,5 & 68,0 & 168,25 & 24,0 & 41,15 & 92,25 & - \\
\hline DP & 7,6 & 10,55 & 4,80 & 3,22 & 9,22 & 2,60 & - \\
\hline
\end{tabular}

Legenda: * = Suplementação de $\mathrm{O}_{2}$ em $4 \mathrm{~W}$ e $5 \mathrm{~W}$; $\mathrm{SpO}_{2}$ inicial: $80 \%$ e $80 \%$; tempo de suplementação: 3 minutos e 1,5 minutos e SpO $\mathrm{final}_{2}$ $98 \%$ e $96 \%$, respectivamente.

Fonte: Dados da pesquisa. 
Tabela 2 - Relação entre carga obtida no $L A(W)$ e $V E F_{1}(\%)$, a potência (W) obtida abaixo do LA, no LA e acima do LA nos pacientes estudados

\begin{tabular}{cccccc}
\hline Pacientes & Carga LA (W) & VEF $_{\mathbf{1}}(\%)$ & Abaixo do LA & LA & Acima do LA \\
\hline $\mathbf{1}$ & 4 & 36 & - & 4 & 5 \\
$\mathbf{2}$ & 20 & 42 & 15 & 20 & 25 \\
$\mathbf{3}$ & 4 & 27 & - & 4 & 5 \\
$\mathbf{4}$ & 4 & 32,5 & - & 4 & 5 \\
$\mathbf{5}$ & 50 & 55,2 & 45 & 50 & 55 \\
$\mathbf{6}$ & 30 & 44 & 25 & 30 & 35 \\
$\mathbf{7}$ & 10 & 50,5 & 5 & 10 & 15 \\
$\mathbf{8}$ & 4 & 42 & - & 4 & 5 \\
\hline Média & $\mathbf{1 5 , 7}$ & $\mathbf{3 9 , 8}$ & $\mathbf{2 2 , 5}$ & $\mathbf{1 5 , 7}$ & $\mathbf{1 8 , 7}$ \\
\hline DP & $\mathbf{1 6 , 8}$ & $\mathbf{9 , 0}$ & $\mathbf{1 7}$ & $\mathbf{1 6 , 8}$ & $\mathbf{1 8 , 4}$ \\
\hline
\end{tabular}

Legenda: - = carga indeterminada, pois os respectivos pacientes apresentaram inclinação positiva no gráfico do comportamento da FC na intensidade mínima do cicloergômetro (4W); LA = limiar de anaerobiose.

Fonte: Dados da pesquisa.

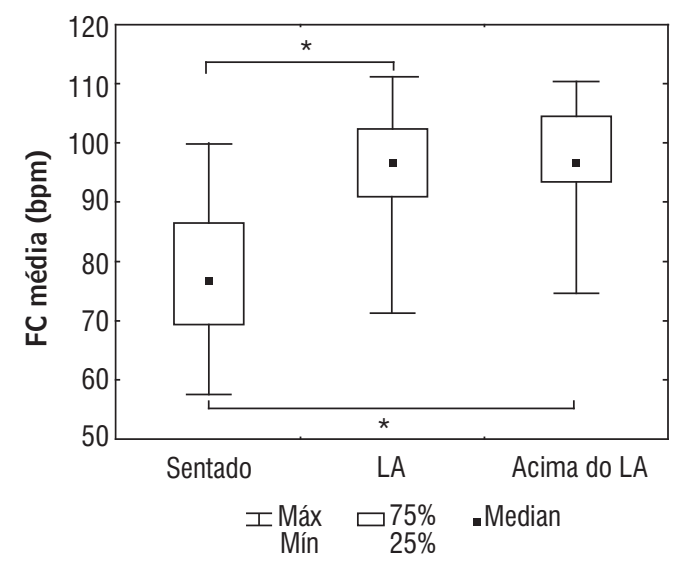

Figura 1 - Valores de FCMed (bpm) obtidos em repouso sentado, nas intensidades do LA e acima do LA Legenda: $L A=$ limiar de anaerobiose; ${ }^{*}=p<0,05$. Fonte: Dados da pesquisa.

Podemos verificar, contudo, que na condição de repouso, ao contrário da FCMed, foram registrados valores significativamente maiores que os obtidos durante o exercício (no LA e acima do LA), não havendo diferença estatística entre esses últimos.

Comportamento semelhante foi verificado para o índice RMSM (Figura 3), no qual os valores obtidos durante o EF dinâmico (no LA e acima do LA)

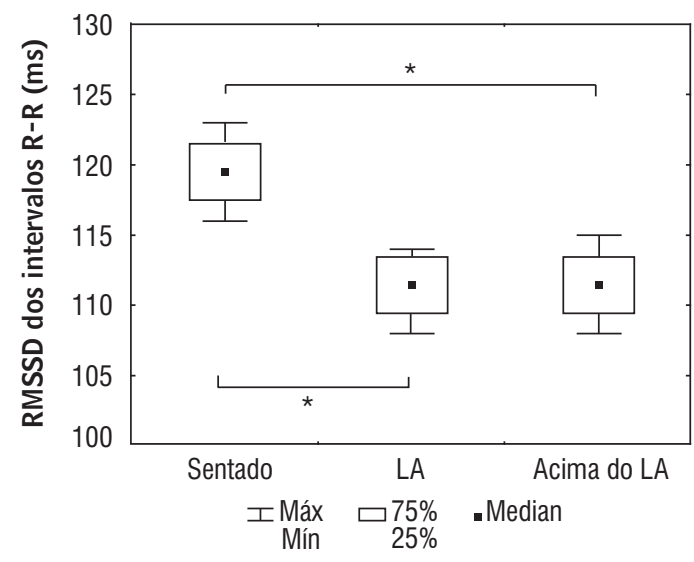

Figura 2 - Valores do índice RMSSD (ms) obtido em repouso sentado e nas velocidades do $L A$ e acima do $L A$ Legenda: $\mathrm{LA}=$ limiar de anaerobiose; ${ }^{*}=p<0,05$. Fonte: Dados da pesquisa.

decresceram significativamente em relação à condição de repouso.

\section{Discussão}

A DPOC promove limitações ventilatórias e disfunção muscular periférica, causando quadros de 


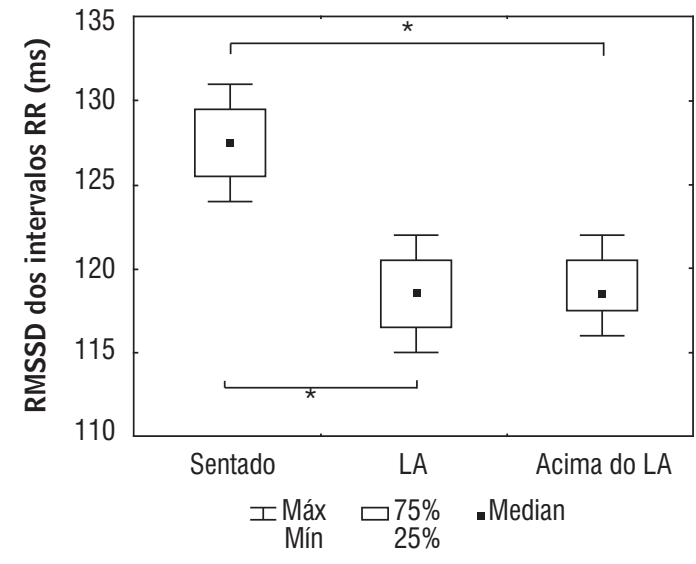

Figura 3 - Valores dos índices RMSM (ms) obtido em repouso sentado e nas velocidades do LA e acima do LA Legenda: $L A=$ limiar de anaerobiose; ${ }^{*}=p<0,05$.

Fonte: Dados da pesquisa.

dispneia e progressivo descondicionamento físico. Ainda nesse sentido, temos que o LA revela a capacidade física individual, servindo de parâmetro para a prescrição de $\mathrm{EF}(8,18)$.

0 valor da potência em que foi obtido o LA variou entre os pacientes estudados, apesar da semelhança da amostra em idade e no índice $\mathrm{VEF}_{1}(<60 \%$ do predito). Para os pacientes $1,3,4$ e 8, não foi possível determinar o nível de esforço em que ocorreu o LA, uma vez que na potência inicial de $4 \mathrm{~W}$, já se pôde observar inclinação positiva da FC. Semelhante resultado foi apresentado por Silva et al. (19), ao estudarem pacientes com DPOC grave, embora com metodologia diferente, não puderam observar em todos os pacientes a determinação precisa do LA, uma vez que, esses pacientes apresentaram lactacidemia precoce a baixas cargas de trabalho.

Foi possível observar, ainda, que o paciente 5 foi aquele que obteve maior índice de $\mathrm{VEF}_{1}$ e maior potência no LA (50W), o que, de acordo com o estudo de Marães (20), corresponde ao LA de indivíduos saudáveis e sedentários. Do mesmo modo, os demais pacientes que apresentaram o LA em intensidades diferentes da mínima ( $4 \mathrm{~W}$ ) também obtiveram maiores $V_{E F}$. A exceção foi o paciente 2, que, apesar de ter $\mathrm{VEF}_{1}$ de $42 \%$ do predito (índice semelhante ao do paciente 8), apresentou o LA na carga de 20W. Alguns trabalhos têm mostrado que nem sempre menores valores de $\mathrm{VEF}_{1}$ indicam maior limitação funcional, fato que pode ser atribuído também ao grau de disfunção muscular periférica (21).

Por outro lado, os pacientes com menores valores de $\mathrm{VEF}_{1}$ apresentaram o LA em potências baixas. Esses resultados estão de acordo com os de outros relatos na literatura $(8,22)$. Dessa forma, maior grau de obstrução promove menor ventilação, que, por sua vez, reflete em menor tolerância ao EF. Na realidade, as modificações na resistência e na força dos músculos esqueléticos periféricos induzidas pelas alterações das trocas gasosas são responsáveis pelas limitações da capacidade física (23). No entanto, a diminuição de força e resistência muscular mostrou-se não ser causada apenas em decorrência das alterações respiratórias, mas também em virtude de um processo adaptativo estrutural pelo qual as fibras musculares esqueléticas periféricas, antes com características predominantemente oxidativas (tipo I), passam agora produzir a energia necessária para sua contração através do metabolismo anaeróbio lático (tipo IIb/x) (24), agravando ainda mais o descondicionamento físico. Desses resultados, permite-se especular que os pacientes com DPOC, dos quais não foram possíveis as determinações do LA, possuam reduzida capacidade física a ponto de estarem vivendo em estado de anaerobiose ou muito próximo a ele.

Por outro lado, na tentativa de minimizar as perdas musculares advindas com o curso da doença, tem sido amplamente prescrito o treinamento físico aeróbio (25). Porém, sabe-se que os pacientes com doença respiratória crônica são principalmente limitados pela insuficiência respiratória antes de atingir a frequência cardíaca máxima ou mudanças nos níveis de lactato. Sendo assim, para esses pacientes, atividades acima de $60 \%$ da capacidade pico são empiricamente consideradas suficientes para provocar um efeito de treinamento (26). Bjorgen (27), comparando o treinamento físico em alta e baixa intensidade, observou melhora das variáveis fisiológicas no treinamento de alta intensidade. Além disso, Silva et al. (19), ao estudarem indivíduos com DPOC, constataram melhora na capacidade aeróbia, na tolerância ao EF e, consequentemente, no aumento da independência dos pacientes quando empregaram um protocolo de treinamento físico com intensidades próximas ao LA.

Já os valores médios da FC se apresentaram maiores no LA e acima do LA em relação à situação sentada, característica atribuída ao predomínio da atividade simpática no nodo sinoatrial, frente ao estresse recebido $(28,29)$. 
Analisando os valores médios dos índices temporais RMSM e RMSSD, pôde-se observar que eles foram menores comparando as situações de repouso e intensidade do LA e se mantiveram na intensidade acima do LA. Essa diminuição nos índices de VFC no esforço dinâmico em relação ao repouso se deve à menor atuação parassimpática no controle da FC, com predomínio da atividade simpática (30). Alonso (28) ainda especula que valores similares dos índices de VFC entre intensidades acima e no LA são resultantes da intensificação nervosa simpática no coração, que promove uma intensa taquicardia, a ponto de estagnar a depressão da VFC.

Nesse sentido, podemos supor que a capacidade física desses indivíduos diminui, uma vez que não foi possível determinar o LA da metade da amostra estudada.

\section{Conclusão}

De acordo com os resultados obtidos, podemos concluir que a VFC dos pacientes com DPOC apresentou-se reduzida durante o EF dinâmico em relação ao repouso enquanto ocorreu elevação contínua da FC.

Além disso, verificamos que o os pacientes estudados apresentaram um severo descondicionamento físico, reafirmado pela impossibilidade em determinar o LA em 50\% da amostra.

\section{Referências}

1. Celli BR, Macnee W, ATS/ERS Task Force. Standards for the diagnosis and treatment of patients with COPD: a summary of the ATS/ERS position paper. Eur Respir J. 2004;23(6):932-46. doi:10.1183/09031936.04.00 014304 .

2. McAnulty RJ. Models and approaches to understand the role of airway remodelling in disease. Pulm Pharmacol Ther. 2011;24(5):478-86. doi:10.1016/j. pupt.2011.07.005.

3. Hartman JE, Boezen HM, de Greef MH, Bossenbroek L, ten Hacken NH. Consequences of physical inactivity in chronic obstructive pulmonary disease. Expert Rev Respir Med. 2010;4(6):735-45. doi:10.1586/ ers.10.76.
4. Miravitlles M, Naberan K, Cantoni J, Azpeitia A. Socioeconomic status and health-related quality of life of patients with chronic obstructive pulmonary disease. Respiration. 82(5):402-8. doi:10.1159/000328766.

5. Marín Royo M, Pellicer Císcar C, González Villaescusa C, Bueso Fabra MJ, Aguar BAC, Rodríguez AL, et al. Physical activity and its relationship with the state of health of stable COPD patients. en representación del grupo EPOC de la Sociedad Valenciana de Neumología (SVN). Arch Bronconeumol. 2011;47(7):33542. doi:10.1016/j.arbres.2011.03.004.

6. Baltzan MA, Scott AS, Wolkove N, Bailes S, Bernard $\mathrm{S}$, Bourbeau J, et al. Fatigue in COPD: prevalence and effect on outcomes in pulmonary rehabilitation. Canadian COPD Pulmonary Rehabilitation Research Group. Chron Respir Dis. 2011;8(2):119-28. PMid:21430090.

7. Scott AS, Baltzan MA, Fox J, Wolkove N. Success in pulmonary rehabilitation in patients with chronic obstructive pulmonary disease. Can Respir J. 2010;17(5):219-23. PMid:21037996.

8. Casaburi R. Skeletal muscle dysfunction in chronic obstructive pulmonary disease. Med. Sci. Exerc. 2001;33(7 Suppl):S662-70. doi:10.1097/ 00005768-200107001-00004.

9. Cooper CB. Exercise in chronic obstructive pulmonary disease: aerobic exercise prescription. Med Sci Sports Exerc. 2001;33(7 Suppl):S671-9. doi:10.1097/00005768-200107001-00005.

10. Dourado VZ, Godoy I. Recondicionamento muscular na DPOC: principais intervenções e novas tendências. Rev Bras Med Esporte. 2004;10(4). doi:10.1590/ S1517-86922004000400010.

11. Vogiatzis I, Williamson AF, Miles J, Taylor IK. Physiological response to moderate exercise workloads in Pulmonary Rehabilitation Program in patients with varying degrees of airflow obstruction. Chest. 1999; 116(5):1200-7. doi:10.1378/chest.116.5.1200.

12. Garcia S, Rocha M, Pinto PMF, Lopes A, Bárbara C. Inspiratory muscle training in COPD patients. Rev Port Pneumol. 2008;14(2):177-94. PMid:18363017.

13. Troosters T, Gosselink R, Decramer M. Exercise training in COPD: how to distinguish responders from nonresponders. J Cardiopulm Rehabil. 2001;21(1):10-7. doi:10.1097/00008483-200101000-00004. 
14. Wasserman DH, Whipp BJ. Coupling of ventilation to pulmonary gas exchange during nonsteady-state work in men. J Appl Physiol. 1983;54(2):587-93. PMid:6833054.

15. Davis JA, Whipp BJ, Lamarra N, Huntsman DJ, Frank $\mathrm{MH}$, Wasserman K. Effect of ramp slope on determination of anaerobic parameters from the ramp exercise test. Med Sci Sports Exerc. 1982;14(5):339-43. doi:10.1249/00005768-198205000-00005.

16. Higa MN, Silva E, Neves VF, Catai AM, Gallo L Jr, Silva de Sá MF. Comparison of anaerobic threshold determined by visual and mathematical methods in healthy women. Braz J Med Biol Res. 2007;40(4):501-8.

17. Borg G. Borg's perceived exertion and pain scales. Champaign: Human Kinects, 1998. p. 49-52.

18. O'Donnell DE. Ventilatory limitations in chronic obstructive pulmonary disease. Med Sci Sports Exerc. 2001;33(7Suppl):S647-55.

19. Silva AB, Di Lorenzo VAP, Sampaio LMM, Jamami M, Costa D, Baldissera V. A influência do treinamento físico (TF) sobre as respostas cardiorrespiratórias e a lactacidemia em pacientes com doença pulmonar obstrutiva crônica (DPOC). Rev Bras Ativid Física \& Saúde. 2003;8(1):30-7.

20. Marães VRFS, Silva E, Catai AM, Gallo L Jr., Oliveira L, et al. Avaliação da capacidade física de pacientes com doença pulmonar obstrutiva crônica de grau leve e moderado a partir da resposta da freqüência cardíaca: relato de caso. Rev Soc Cardiol. Estado de São Paulo. 2000;10:6-15.

21. Beier J, Beeh KM. Long-acting $\beta$-adrenoceptor agonists in the management of COPD: focus on indacaterol. Int J Chron Obstruct Pulmon Dis. 2011;6:237-43.

22. Casaburi R, Porszasz J, Burns MR, ER Carithers, Chang RS, Cooper CB. Physiologic benefits of exercise training in rehabilitation of patients with severe COPD. Am J Respir Crit Care Med. 1997;155(5):1541-51.

23. Gagnon P, Saey D, Vivodtzev I, Laviolette L, Mainguy $\mathrm{V}$, Millot J, et al. Impact of preinduced quadriceps fatigue on exercise response in chronic obstructive pulmonary disease and healthy subjects. J Appl Physiol. 2009;107(3):832-40.
24. Gosker HR, Wouters EF, van der Vusse GJ, Schols AM. Skeletal muscle dysfunction in chronic obstructive pulmonary disease and chronic heart failure: underlying mechanisms and therapy perspectives. Am J Clin. Nutr. 2000;71(5):1033-47.

25. Dolmage TE, Goldstein RS. Effects of one-legged exercise training of patients with COPD. Chest. 2008;133(2):370-6.

26. van Helvoort HA, de Boer RC, van de Broek L, Dekhuijzen R, Heijdra YF. Exercises commonly used in rehabilitation of patients with chronic obstructive pulmonary disease: cardiopulmonary responses and effect over time. Arch Phys Med Rehabil. 2011;92(1):111-7.

27. Bjørgen S, Helgerud J, Husby V, Steinshamn S, Richadson RR, Hoff J. Aerobic high intensity one-legged interval cycling improves peak oxygen uptake in chronic obstructive pulmonary disease patients; no additional effect from hyperoxia. Int J Sports Med. 2009;30(12):872-8.

28. Hofmann P, Pokan R. Value of the application of the heart rate performance curve in sports. Int J Sports Physiol Perform. 2010;5(4):437-47.

29. Alonso DO, Forjaz CL, Rezende LO, Braga AM, Barretto ACP, et al. Comportamento da freqüência cardíaca e da sua variabilidade durante as diferentes fases do exercício físico progressivo máximo. Arq Bras Cardiol. 1998;71(6):787-92.

30. Crisafulli A, Salis E, Pittau G, Lorrai L, Tocco F, et al. Modulation of cardiac contractility by muscle metaboreflex following efforts of different intensities in humans. Am J Physiol Heart Circ Physiol. 2006; 291(6): H3035-H3042.

Recebido: $11 / 08 / 2011$ Received: 08/11/2011

Aprovado: 03/06/2012 Approved: 06/03/2012 